\title{
The International Congress on Hypergraphs, Graphs and Designs - HyGraDe 2017
}

This issue of ADAM - The Art of Discrete and Applied Mathematics offers a collection of papers presented at the International Congress on Hypergraphs, Graphs and Designs HyGraDe 2017, which took place in Sant'Alessio Siculo, Sicily, Italy, June 20 - 24, 2017. HyGraDe 2017 was conceived with the idea of celebrating the 70th birthday of Mario Gionfriddo, a Sicilian mathematician who has devoted his long and successful career to the study of graphs, hypergraphs and designs.

The conference HyGraDe 2017 was organized by Francesco Belardo (University of Naples Federico II) and Giovanni Lo Faro (University of Messina), both serving as chair of the Organizing Committee, Luca Giuzzi (University of Brescia), Enzo M. Li Marzi (University of Messina), Lorenzo Milazzo (University of Catania), Salvatore Milici (University of Catania) and Antoinette Tripodi (University of Messina). The Scientific Committee consisted of Marco Buratti (University of Perugia), Giovanni Lo Faro, Guglielmo Lunardon (University of Naples Federico II) and Martin Milanič (University of Primorska).

The conference brought together scientists working in different disciplines of Combinatorics. There were 85 participants from 13 different countries, 10 invited talks and 35 contributed talks touching on the latest developments in the corresponding research areas. The invited speakers were Richard Brualdi (University of Winsconsin), Marco Buratti (University of Perugia), Charlie Colbourn (Arizona State University), Klavdjia Kutnar (University of Primorska), Josef Lauri (University of Malta), Curt Lindner (University of Auburn), Dragan Marušič (University of Primorska), Alex Rosa (McMaster University), Zsolt Tuza (Hungarian Academy of Sciences) and Vitaly Voloshin (Troy University). Mario Gionfriddo was the honoured participant at the congress. Among the invited speakers, six of them have been Mario's co-authors in several scientific papers.

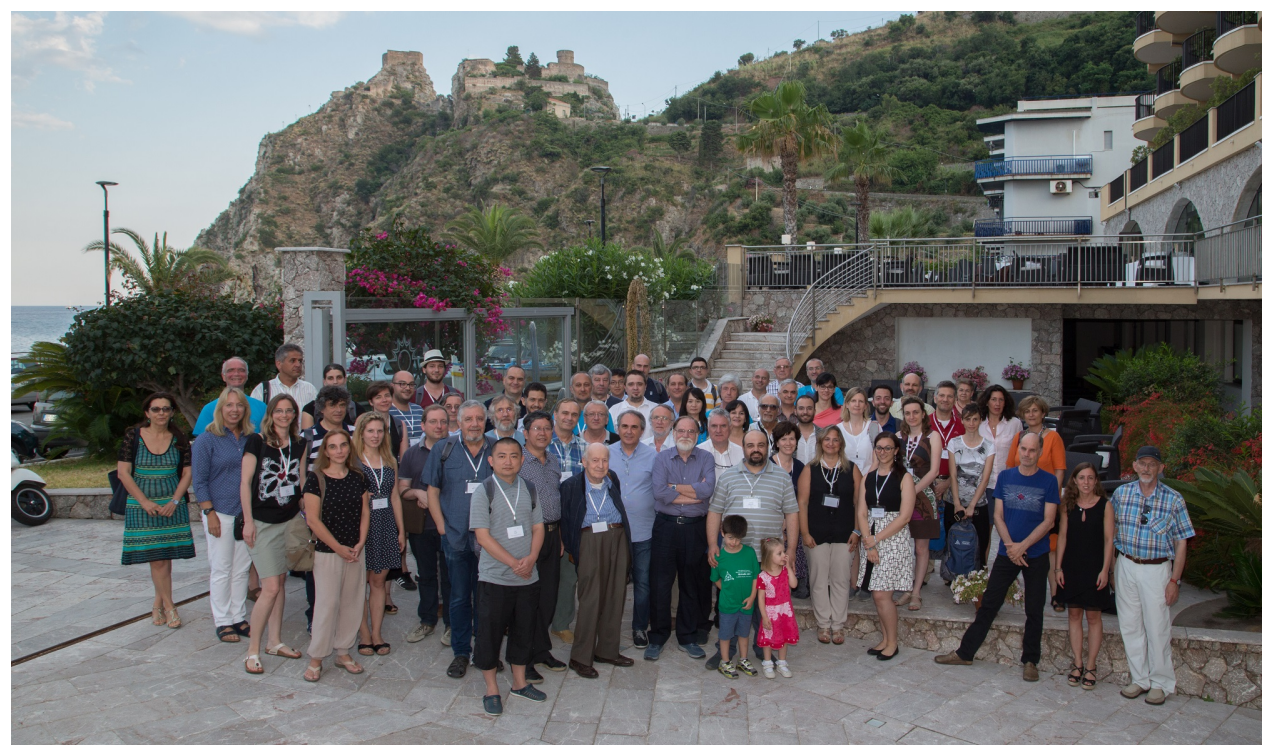

The Conference Photo 
The Organizing Committee thanks all who contributed to the successful organization of this event. In particular the Organizing Committee is grateful to the sponsors of this conference: Universities of Catania, Messina and Naples Federico II, the Department of Mathematics and Informatics of Catania, the Department MIFT of Messina, the INDAMGNSAGA and the non-profit association Combinatorics 2014. We also recognize the kind support of the University of Primorska, the Department of Mathematics and Applications "R. Caccioppoli" of Naples and the Accademia Peloritana dei Pericolanti.

We are grateful to the Editors-in-Chief, Professors Tomo Pisanski and Dragan Marušič, for this issue of ADAM. We also thank all the colleagues who have participated in this initiative and the referees who have reviewed the papers. We would like to mention that a special issue of the Sicilian scientific journal Atti Accademia Peloritana dei Pericolanti - AAPP contains other contributions from the participants of HyGraDe 2017. The special issue of AAPP devoted to HyGraDe 2017 can be accessed at http: / / cab . unime. it / journals/index.php/AAPP/issue/view/Vol96_supplement2.

Francesco Belardo

Guest Editor 